\title{
Chapter 1 \\ Multi-Level Quasi-Newton Methods for the Partitioned Simulation of Fluid-Structure Interaction
}

Joris Degroote, Sebastiaan Annerel and Jan Vierendeels

\begin{abstract}
In previous work, Fourier stability analyses have been performed of Gauss-Seidel iterations between the flow solver and the structural solver in a partitioned fluid-structure interaction simulation. These analyses of the flow in an elastic tube demonstrated that only a number of Fourier modes in the error on the interface displacement are unstable. Moreover, the modes with a low wave number are most unstable and these modes can be resolved on a coarser grid. Therefore, a new class of quasi-Newton methods with more than one grid level is introduced. Numerical experiments show a significant reduction in run time.
\end{abstract}

\subsection{Introduction}

Partitioned fluid-structure interaction (FSI) simulation techniques solve the flow equations and the structural equations separately. Strongly coupled partitioned techniques enforce the equilibrium of the stress and velocity (or displacement) on the fluid-structure interface in each time step. Several strongly coupled partitioned techniques are able to couple 'black-box' solvers, for example the Interface Block Quasi-Newton technique with an approximation for the Jacobians from LeastSquares models (IBQN-LS) [5] and the Interface Quasi-Newton technique with an

Joris Degroote

Ghent University, Department of Flow, Heat and Combustion Mechanics, Sint-Pietersnieuwstraat 41, 9000 Ghent, Belgium, e-mail: Joris.Degroote@UGent.be

Sebastiaan Annerel

Ghent University, Department of Flow, Heat and Combustion Mechanics, Sint-Pietersnieuwstraat 41, 9000 Ghent, Belgium, e-mail: Sebastiaan.Annerel@UGent.be

Jan Vierendeels

Ghent University, Department of Flow, Heat and Combustion Mechanics, Sint-Pietersnieuwstraat 41, 9000 Ghent, Belgium, e-mail: Jan.Vierendeels@UGent.be 
approximation for the Inverse of the Jacobian from a Least-Squares model (IQNILS) [2].

The coupling iterations of strongly coupled partitioned techniques can suffer from stability issues. Degroote et al. [1,3] performed a Fourier decomposition of the error on the interface displacement during Gauss-Seidel iterations. These analyses show that only a fraction of all Fourier modes is unstable, notably the modes with a low wave number. These unstable modes can be resolved on a coarser grid. Therefore, the new Multi-Level (ML) coupling techniques presented in this work use more than one grid level, each with a different number of grid points. Below, Multi-Level IQN-ILS (ML-IQN-ILS) is derived from IQN-ILS; Multi-Level IBQNLS (ML-IBQN-LS) can be derived from IBQN-LS in a similar way.

\subsection{Governing equations}

A Dirichlet-Neumann decomposition of the fluid-structure interaction problem is applied. Consequently, the flow and structural solver can be represented by the functions

$$
\mathbf{y}=\mathscr{F}(\mathbf{x}) \quad \text { and } \quad \mathbf{x}=\mathscr{S}(\mathbf{y}),
$$

respectively. The vector $\mathbf{x}$ represents the displacement of all nodes on the interface and the vector $\mathbf{y}$ represents the stress on all edges/faces of the interface.

As the multi-level coupling algorithms use several grid levels for the flow equations and the structural equations, data has to be interpolated between different discretizations of the fluid-structure interface. Even though the discretization of the interface inside the flow solver and the structural solver depends on the grid level, all operations of the coupling algorithm are performed on a single grid - the so-called 'coupling grid' (see Fig. 1.1) — as the interpolation is hidden inside the solvers. In this work, this coupling grid is identical to the interface discretization of the finest fluid grid.

Fig. 1.1 Abstract representation of coarse (light) and fine (dark) grid levels in the structural solver (left) and the flow solver (right), together with the coupling grid (centre)

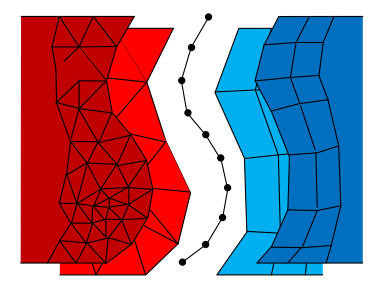

Local radial basis function interpolation is used on the interface [6]. If, for example, a stress component has to be interpolated from a fluid grid to the coupling grid, then three steps are performed for each point of the coupling grid. First, a given number of points on the fluid grid with the smallest Euclidean distance to the point of the coupling grid are selected. Then, the interpolation coefficients are calculated, 
using $\phi(z)=(1-z)_{+}^{4}(4 z+1)$ as basis function [6], with $z$ the Euclidean distance divided by the radius. The plus-sign behind the first term denotes that this term is zero if $1-z<0$ such that $\phi$ has a compact support. In this work, these first two steps are performed only once at the beginning of the simulation. Finally, the stress component is interpolated, using the interpolation coefficients from the second step.

\subsection{Coupling algorithm}

In this section, the standard IQN-ILS algorithm [2] with one grid level is first explained and subsequently extended to the ML-IQN-ILS algorithm. A prime denotes the Jacobian matrix of a function and a hat refers to an approximation. The output of a solver is indicated with a tilde. The grid level is indicated with a subscript $i$, with the first grid level the coarsest one and the $g^{\text {th }}$ grid level the finest one. The coupling iteration within time step $n+1$ is denoted with a superscript $k$.

The standard IQN-ILS coupling technique solves the FSI problem reformulated as a set of nonlinear equations in the interface's displacement

$$
\mathscr{R}(\mathbf{x})=\mathscr{S} \circ \mathscr{F}(\mathbf{x})-\mathbf{x}=\mathbf{0}
$$

by means of quasi-Newton iterations

$$
\mathbf{x}^{k+1}=\mathbf{x}^{k}+\widehat{\left(\mathscr{R}^{\prime k}\right)^{-1}}\left(-\mathbf{r}^{k}\right),
$$

using an approximation for the product of the inverse of the Jacobian matrix with the vector $-\mathbf{r}^{k}$. The residual is calculated as $\mathbf{r}^{k}=\mathscr{R}\left(\mathbf{x}^{k}\right)=\mathscr{S} \circ \mathscr{F}\left(\mathbf{x}^{k}\right)-\mathbf{x}^{k}=\tilde{\mathbf{x}}^{k}-\mathbf{x}^{k}$. The coupling iterations in the time step have converged when $\left\|\mathbf{r}^{k}\right\|_{2} \leq \varepsilon_{o}$ with $\varepsilon_{o}$ the convergence tolerance.

The vector $-\mathbf{r}^{k}$ is the difference between the desired residual, i.e. $\mathbf{0}$, and the current residual $\mathbf{r}^{k}$ and it is further denoted as $\Delta \mathbf{r}=\mathbf{0}-\mathbf{r}^{k}=-\mathbf{r}^{k}$. The matrixvector product is approximated using information obtained during the previous quasi-Newton iterations. To that end, the matrices

$$
\begin{aligned}
\mathbf{V}^{k} & =\left[\begin{array}{llll}
\Delta \mathbf{r}^{k-1} & \Delta \mathbf{r}^{k-2} & \ldots \Delta \mathbf{r}^{1} \Delta \mathbf{r}^{0}
\end{array}\right] \\
\mathbf{W}^{k} & =\left[\begin{array}{llll}
\Delta \tilde{\mathbf{x}}^{k-1} & \Delta \tilde{\mathbf{x}}^{k-2} & \ldots \Delta \tilde{\mathbf{x}}^{1} \Delta \tilde{\mathbf{x}}^{0}
\end{array}\right]
\end{aligned}
$$

are constructed, with $\Delta \mathbf{r}^{m-1}=\mathbf{r}^{m}-\mathbf{r}^{m-1}$ and $\Delta \tilde{\mathbf{x}}^{m-1}=\tilde{\mathbf{x}}^{m}-\tilde{\mathbf{x}}^{m-1}(m=1, \ldots, k)$. These matrices contain the differences between, respectively, residual vectors and outputs of the structural solver in consecutive coupling iterations. The change of the residual vector $(\Delta \mathbf{r})$ is decomposed as a linear combination of the differences between previous residual vectors

$$
\Delta \mathbf{r} \approx \mathbf{V}^{k} \mathbf{c}^{k}
$$


with $\mathbf{c}^{k}$ the coefficients of the decomposition. As the matrix $\mathbf{V}^{k}$ normally contains more rows than columns, the decomposition coefficients $\mathbf{c}^{k}$ are calculated by solving a least-squares problem, using a $\mathrm{QR}$-decomposition of $\mathbf{V}^{k}$. The change of the output of the structural solver $(\Delta \tilde{\mathbf{x}})$ that corresponds to the change of the residual $(\Delta \mathbf{r})$ is subsequently calculated as a linear combination of the previous changes of the output of the structural solver $\left(\Delta \tilde{\mathbf{x}}^{m-1}, m=1, \ldots, k\right)$, analogous to Eq. (1.5), giving

$$
\Delta \tilde{\mathbf{x}}=\mathbf{W}^{k} \mathbf{c}^{k}
$$

From $\mathbf{r}^{k}=\tilde{\mathbf{x}}^{k}-\mathbf{x}^{k}$, it follows that $\Delta \mathbf{r}=\Delta \tilde{\mathbf{x}}-\Delta \mathbf{x}$, so

$$
\Delta \mathbf{x}=\mathbf{W}^{k} \mathbf{c}^{k}-\Delta \mathbf{r}
$$

This can be interpreted as a procedure to calculate the approximation for the product of the inverse of the Jacobian matrix with the vector $\Delta \mathbf{r}=-\mathbf{r}^{k}$.

Algorithm 1 shows the Multi-Level IQN-ILS (ML-IQN-ILS) algorithm. Line 6 to 13 are the standard IQN-ILS algorithm as described above. Around the standard algorithm, an additional loop over the grid levels is added (line 3). First, the coupled solution is calculated on the coarsest grid level. Then, the solution is transferred to the next grid level on line 15 to 19 , followed by coupling iterations on that grid level. These steps are subsequently repeated for all grid levels until the solution on the finest grid has been found. The transfer of the displacement to the following grid level provides the initial value for the coupling iterations on that grid level. The variable $\ell$ (line 1 ) ensures that at least one coupling iteration is performed on each grid level.

Because the coupling algorithm operates on a coupling grid, the difference between $\mathbf{r}$ and $\tilde{\mathbf{x}}$ in consecutive coupling iterations is always interpolated to a fixed number of grid points, regardless of the current grid level. As a result, the modes that have been generated on a coarse grid level can be used to accelerate the coupling iterations on the finer grid levels. The same least-squares model is used for all grid levels so the number of columns in the matrices $\mathbf{V}^{k}$ and $\mathbf{W}^{k}$ increases on each grid level. Because the matrices $\mathbf{V}^{k}$ and $\mathbf{W}^{k}$ have to contain at least one column, a relaxation with a constant factor $\omega$ (line 7) is performed in the second coupling iteration of each time step. The counter $k$ is only set to zero at the beginning of the time step (line 1) and not when the coupling iterations on a following grid level start. As a result, the relaxation step is only performed on the coarsest grid level. However, it should be noted that the difference between $\mathbf{r}$ and $\tilde{\mathbf{x}}$ in the last coupling iteration on a certain grid level $i$ and the first coupling iteration on the following grid level $i+1$ should not be added to $\mathbf{V}^{k}$ and $\mathbf{W}^{k}$, as these differences are biased because the terms have been calculated on two different grid levels.

Line 21 to 23 show that synchronization is necessary at the end of the time step. Once the solution has been found on the finest grid level, all degrees of freedom on the coarser grid levels have to be corrected. 


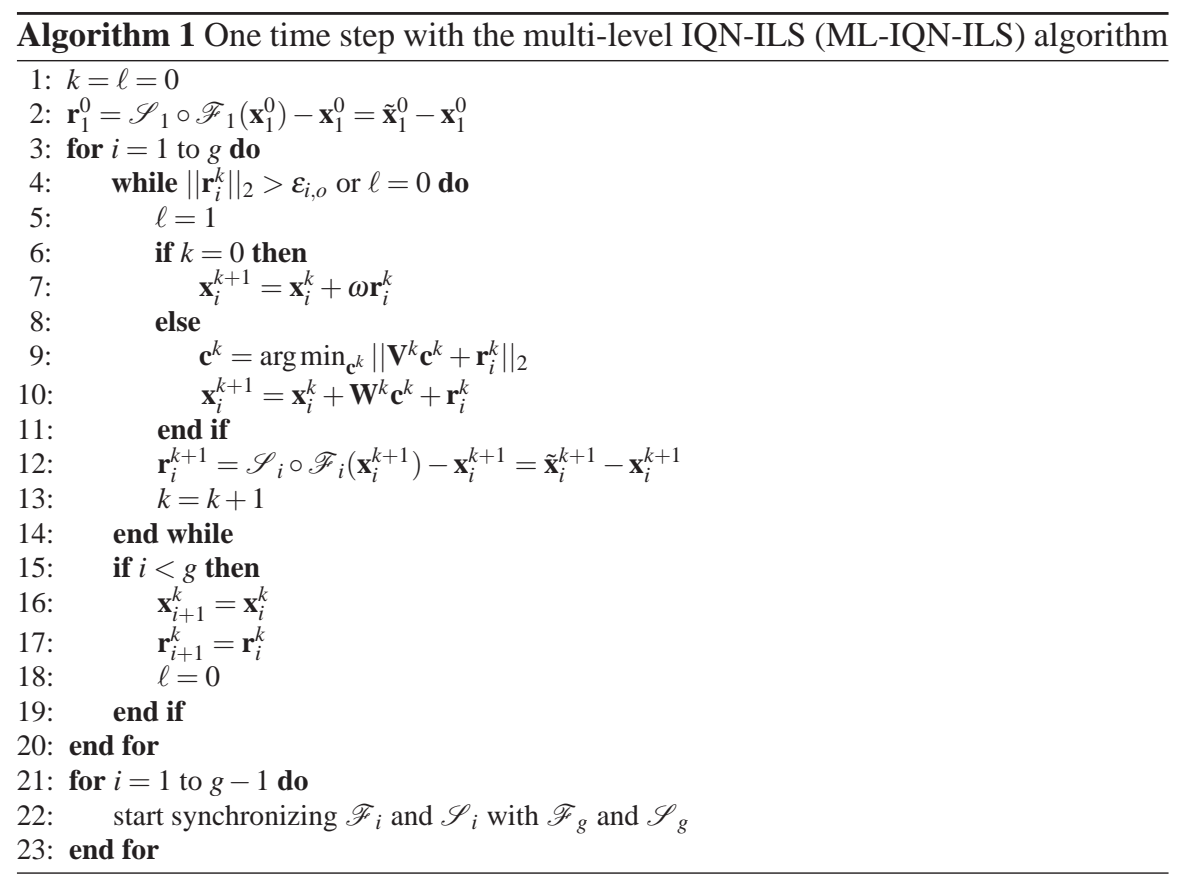

\subsection{Numerical results}

To assess the performance of the multi-level algorithms, the propagation of a pressure wave in a straight flexible tube is simulated [4]. Pressure contours on the fluidstructure interface are shown in Figure 1.2.

Table 1.1 lists the number of coupling iterations per time step and per grid level, averaged over the entire simulation, and the relative duration of the simulations. The coarse grid level contains 34944+1824 degrees of freedom for the flow and the structure, respectively, while the fine grid level contains $2247168+28032$ degrees of freedom. In the simulation with two grid levels, the number of coupling iterations on the fine grid is reduced by approximately $50 \%$ compared to a simulation with a fine grid only. As the cost of the coupling iterations on the coarse grid level is relatively small, the duration of the simulation also decreases by approximately $50 \%$.

Fig. 1.2 Pressure contours (in $\mathrm{Pa}$ ) on the fluid-structure interface after $10^{-3} \mathrm{~s}$ (left), $5 \times 10^{-3}$ s (centre) and $9 \times$ $10^{-3} \mathrm{~s}$ (right)

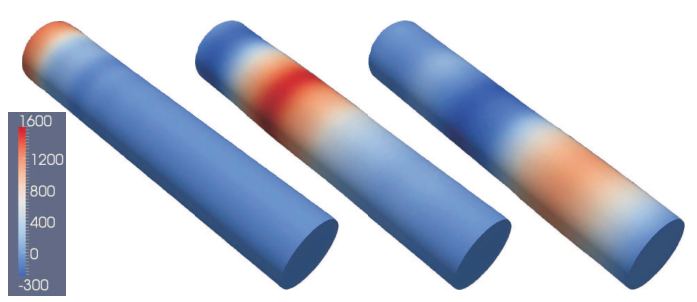


Table 1.1 Comparison between one and two grid levels for the propagation of a pressure wave in a $3 \mathrm{D}$ tube

\begin{tabular}{|c|c|c|c|}
\hline Algorithm & Coarse iters & Fine iters & Duration \\
\hline IQN-ILS & - & 13.2 & 1.9 \\
\hline ML-IQN-ILS & 12.1 & 7.0 & 1.0 \\
\hline IBQN-LS & - & 13.3 & 2.0 \\
\hline ML-IBQN-LS & 12.5 & 6.6 & 1.0 \\
\hline
\end{tabular}

\subsection{Conclusion}

Stability analyses on Gauss-Seidel coupling iterations demonstrated that the Fourier modes with a low wave number in the error on the interface displacement are most unstable. The new multi-level algorithms resolve these modes with a low wave number on a coarser grid. The numerical results show that these multi-level algorithms can reduce the duration of a partitioned fluid-structure interaction simulation, if the difference in number of degrees of freedom between the grid levels is sufficient.

Acknowledgements J. Degroote gratefully acknowledges a Ph.D. fellowship of the Research Foundation - Flanders (FWO)

\section{References}

[1] Degroote J, Bruggeman P, Haelterman R et al (2008) Stability of a coupling technique for partitioned solvers in FSI applications. Comput Struct 86:22242234

[2] Degroote J, Bathe KJ, Vierendeels J (2009) Performance of a new partitioned procedure versus a monolithic procedure in fluid-structure interaction. Comput Struct 87:793-801

[3] Degroote J, Annerel S, Vierendeels J (2010) Stability analysis of Gauss-Seidel iterations in a partitioned simulation of fluid-structure interaction. Comput Struct 88:263-270

[4] Formaggia L, Gerbeau JF, Nobile F et al (2001) On the coupling of 3D and 1D Navier-Stokes equations for flow problems in compliant vessels. Comput Meth Appl Mech Eng 191:561-582

[5] Vierendeels J, Lanoye L, Degroote J et al (2007) Implicit coupling of partitioned fluid-structure interaction problems with reduced order models. Comput Struct 85:970-976

[6] Wendland H (1995) Piecewise polynomial, positive definite and compactly supported radial functions of minimal degree. Adv Comput Math 4:389-396 